\title{
Actively Secure Two-Party Evaluation of Any Quantum Operation
}

\author{
Frédéric Dupuis $^{1, \star}$, Jesper Buus Nielsen ${ }^{2, \star \star}$, and Louis Salvail ${ }^{3, \star \star \star}$ \\ 1 Institute for Theoretical Physics, ETH Zurich, Switzerland \\ dupuis@phys.ethz.ch \\ ${ }^{2}$ Department of Computer Science, Aarhus University, Denmark \\ jbn@cs.au.dk \\ 3 Université de Montréal (DIRO), QC, Canada \\ salvail@iro.umontreal.ca
}

\begin{abstract}
We provide the first two-party protocol allowing Alice and Bob to evaluate privately even against active adversaries any completely positive, tracepreserving map $\mathscr{F} \in \mathrm{L}\left(\mathcal{A}_{\text {in }} \otimes \mathcal{B}_{\text {in }}\right) \rightarrow \mathrm{L}\left(\mathcal{A}_{\text {out }} \otimes \mathcal{B}_{\text {out }}\right)$, given as a quantum circuit, upon their joint quantum input state $\rho_{\text {in }} \in \mathrm{D}\left(\mathcal{A}_{\text {in }} \otimes \mathcal{B}_{\text {in }}\right)$. Our protocol leaks no more to any active adversary than an ideal functionality for $\mathscr{F}$ provided Alice and Bob have the cryptographic resources for active secure two-party classical computation. Our protocol is constructed from the protocol for the same task secure against specious adversaries presented in [4].
\end{abstract}

\section{Introduction}

We provide the first active-secure two-party protocol for computing on quantum data. We look at a model where Alice and Bob hold an input $\rho_{\text {in }}$ on registers $\mathcal{A}_{\text {in }}$ and $\mathcal{B}_{\text {in }}$, where Alice holds register $\mathcal{A}_{\text {in }}$ and Bob holds $\mathcal{B}_{\text {in }}$. They agree on a completely positive, trace-preserving (CPTP) map $\mathscr{F}$ from registers $\mathcal{A}_{\text {in }} \otimes \mathcal{B}_{\text {in }}$ to registers $\mathcal{A}_{\text {out }} \otimes \mathcal{B}_{\text {out }}$, and they want to compute $\rho_{\text {out }}=\mathscr{F}\left(\rho_{\text {in }}\right)$ such that, at the end of the protocol, Alice is in possession of $\mathcal{A}_{\text {out }}$ and Bob is in possession of $\mathcal{B}_{\text {out }}$. They want to do this in an actively secure manner. Our notion of active security is phrased via simulation, but intuitively it simply guarantees that any cheating Alice, even an infinitely powerful Alice, which might deviate from the protocol, can only affect the output of the protocol by replacing her own input and that she will at any point during the execution of the protocol only hold information which can be computed (efficiently) from either $\rho_{\text {in }}^{\mathcal{A}}$ or $\rho_{\text {out }}^{\mathcal{A}}$. The equivalent condition should hold for Bob.

* Supported by Canada's NSERC Postdoctoral Fellowship Program and by the Swiss National Science Foundation via the National Center of Competence QSIT.

** Partially supported by an European Research Council, Starting Grant, number 279447 and the Danish National Research Foundation and the National Science Foundation of China (under the grant 61061130540) for the Sino-Danish Center for the Theory of Interactive Computation.

${ }^{\star \star \star}$ Supported by Canada's NSERC discovery grant, FREQUENCY(NSERC), the QuantumWorks networks(NSERC), and INTRIQ(FQRNT). 
A simple example of such an $\mathscr{F}$ is the quantum swap, where $\mathcal{A}_{\text {in }}=\mathcal{A}_{\text {out }}=\mathcal{A}$, $\mathcal{B}_{\text {in }}=\mathcal{B}_{\text {out }}=\mathcal{B}$, and $\rho_{\text {out }}^{\mathcal{A}}=\rho_{\text {in }}^{\mathcal{B}}$ and $\rho_{\text {out }}^{\mathcal{B}}=\rho_{\text {in }}^{\mathcal{A}}$. Securely implementing this unitary basically means to do an atomic swap of quantum states, which was shown impossible in [4] even against a restricted class of adversaries called specious. Extra assumptions are therefore needed to get unconditional security. Our way out is to look at a model where the two parties have access to an ideal functionality which allows them to securely do any classical computation on any classical data held jointly by the two parties. In this model we give an unconditionally secure protocol with active security. This is the first such protocol.

Formally, we use the notationally simpler model of [4], but it is easy to see that security in this model implies security in the stand-alone model of [11], as long as the simulator is poly-time. The stand-alone model of [11] allows, inside a secure quantum protocol, to replace a classical ideal functionality by a classical protocol which securely implements that ideal functionality against poly-time quantum adversaries. The result is a protocol secure against poly-time quantum adversaries. This, in particular, implies sequential security, i.e., if a protocol is secure, it remains secure when run in sequence with other secure protocols. Since the secure evaluation of any classical function with security against poly-time quantum adversaries can be done under the assumption that learning with errors is hard [13 11] and under the more general assumption that mixed commitment schemes exist [12], our poly-time simulator provides an active-secure twoparty plain-model protocol for computing on quantum data with security against any poly-time quantum adversaries. This is the first such protocol.

\subsection{Overview of Our Construction}

We reuse many ideas from the protocol provided in [4], which gives a two-party protocol for computing on quantum data securely against so-called specious adversaries. The protocol therein is unconditionally secure given an ideal functionality for classical computation. Specious adversaries are a quantum version of the classical notion of passive adversaries. Technically, a specious adversary is an adversary which is allowed to deviate from the protocol, except that at any step of the protocol it should be able to reconstruct the honest state of the protocol from its current state. This basically allows it to purify itself and not much else.

In the protocol in [4], all wires are encrypted using a Pauli encryption: a qubit $|v\rangle$ is represented as $|V\rangle=\mathrm{X}^{x} \mathrm{Z}^{z}|v\rangle$, where the two uniform key bits $x$ and $z$ are secret-shared between Alice and Bob. For example, to secret-share $x$, Alice will hold a uniformly random bit $x_{A}$ and Bob will hold a uniformly random bit $x_{B}$ such that $x=x_{A} \oplus x_{B}$. All wires are independently encrypted like this, which ensures that intermediate states are perfectly hidden from both parties. Computation is then done "through the encryption". The CPTP map $\mathscr{F}$ is described by a quantum circuit made out of the universal set of gates $\mathcal{U G}$ consisting of gates $\mathrm{X}, \mathrm{Y}, \mathrm{Z}, \mathrm{CNOT}, \mathrm{H}=\frac{1}{\sqrt{2}}\left(\begin{array}{cc}1 & 1 \\ 1 & -1\end{array}\right), \mathrm{P}=\left(\begin{array}{ll}1 & 0 \\ 0 & i\end{array}\right)$, and $\mathrm{R}=\left(\begin{array}{ll}1 & 0 \\ 0 & e^{i \pi / 4}\end{array}\right)$, together with a set of ancilla wires initialized in state $|0\rangle$, and a set of output wires that are discarded (or, in our case, that remain encrypted forever). The protocol evaluates each gate of $\mathscr{F}$ while preserving privacy. Handling the Pauli gates $\mathrm{X}, \mathrm{Y}$ and $\mathrm{Z}$ is easy, as they commute or anti-commute with the encryption operators. 
As an example, assume that the parties want to apply an $\mathbf{X}$ gate to a qubit $|v\rangle$, i.e., compute an encryption of $\left|v^{\prime}\right\rangle=\mathrm{X}|v\rangle$. Since up to an overall phase factor $\mathbf{X Z}=\mathbf{Z X}$, it follows that $\mathbf{X}|V\rangle=\mathbf{X}^{x} \mathbf{Z}^{z}|v\rangle=\mathbf{X}^{x} \mathbf{Z}^{z} \mathbf{X}|v\rangle=\mathbf{X}^{x} \mathbf{Z}^{z}\left|v^{\prime}\right\rangle$. So, the evaluation is simply performed on the encrypted qubit $|V\rangle$ and the key bits $x$ and $z$ are maintained. For the remaining Clifford gates CNOT, $\mathrm{H}$ and $P$ other "commutation" rules with $\mathrm{X}$ and $\mathrm{Z}$ are used. For $\mathrm{H}$, it is used that $\mathrm{HX}=\mathrm{ZH}$ and $\mathrm{HZ}=\mathrm{XH}$, so $\mathrm{H}$ is simply applied to the encrypted qubit, and then the keys $x$ and $z$ are swapped: Alice sets $x_{A}^{\prime}=z_{A}$ and $z_{A}^{\prime}=x_{A}$ and similarly for Bob. This leaves the non-Clifford gate $\mathrm{R}$, where the relation $\mathrm{RX}^{x} \mathrm{Z}^{z}=\mathrm{P}^{x} \mathrm{X}^{x} \mathrm{Z}^{z} \mathrm{R}$ almost does the job, except that it leaves an extra $\mathrm{P}^{x}$. Getting rid of this requires quantum computation and a classical secure two-party computation which computes how the parties should update their key bits. After such a gate-by-gate computation of $U$ on the encrypted qubits, the shares of the keys needed for learning the states on ones own output wires are swapped with the other party in one atomic step, using the ideal functionality for classical secure computation.

The protocol in [4] is actually secure against an active adversary up to the final swap of key bits, as the encryptions of the wires are guaranteed to be perfect, independent of the behavior of the other party, as the parties pick their own shares of the sharings of $x$ and $z$. This means that no party can get any information on any intermediary states, no matter how it deviates. It can, however, easily force the computation to be incorrect, by applying gates to the encrypted states, so the full protocol is not active secure. We note, however, that [4] is secure against what we could call ultimately specious adversaries: Adversaries promising to attack in such a way that both parties always be able to reconstruct the correct state at the end of the protocol, but that can otherwise behave as they want. This follows from the active security in the middle and a theorem in [4] which says that any attack which always allows both parties to obtain the correct output can be simulated given just the output of the corrupted party-basically, there is no way to learn extra information without sometimes irrevocably destroying the state of the other party.

In this paper, we use this observation in a protocol proceeding along the same lines as [4] but where we force the adversary to be ultimately specious. This is done by not only encrypting the wires, but by unconditionally authenticating them. In addition, we commit the parties to their key bits, to allow the recipient to verify the key bits swapped at the end. Since an unconditional quantum authentication code is also an unconditionally secure encryption, we get a protocol with at least the security of [4], but with the added property that an adversary who deviates from the protocol will be caught. More technically, if all checks of the authentication code succeed, then the authenticated values collapse to the correct values. This forces the adversary to either be detected or be ultimately specious. Since we do all the checks of the authentication codes before any key bits are revealed, the case where the adversary is detected can be simulated by simply asking the ideal world to abort too. The case where the adversary is ultimately specious is simulated similarly to [4].

The main technical challenge is then to devise an authentication code with these two properties:

1. It allows to perform computation "through the authentication".

2. It allows to check the authentication code without revealing what is authenticated. 
The first property is important for hiding intermediate values during the computation. The second property is important when we force the adversary to either be detected or ultimately specious: when he is detected, he should learn nothing on the incorrect outputs.

We devise an authentication code with these properties based on the Clifford-based quantum authentication code proposed in [1]. It authenticates a quantum message using a random unitary implementable using gates $\mathrm{X}, \mathrm{Y}, \mathrm{Z}, \mathrm{CNOT}, \mathrm{H}$ and $\mathrm{P}$. The authentication works as follows. In order to satisfy the second property, take a qubit $|v\rangle$ on some wire. Alice prepends $n$ new wires, in the all-zero state $\left|0^{n}\right\rangle$. Then she applies a uniformly random $(n+1)$-bit Clifford operator $A$ to the $n+1$ wires. She then sends the state to Bob, who appends $n$ more wires in the all-zero state and applies a uniformly random $(2 n+1)$-bit Clifford operator $B$ to the $2 n+1$ wires. We can write this as $|V\rangle=B\left(\left|0^{n}\right\rangle \otimes A\left(|v\rangle\left|0^{n}\right\rangle\right)\right)$. The key is $(A, B)$. This authentication can be checked in two different ways. Either, apply $B^{\dagger}$ to the authenticated state and check that the first $n$ wires are all zero. Or, apply $B^{\dagger}$ and then apply $A^{\dagger}$ to the last $n+1$ wires and check that the last $n$ wires are all zero. One way is used for Alice to check that Bob did not change the authenticated value. The other is used by Bob to check Alice. In order to perform these without leakage, we use a 2-party classical ideal functionality as described below.

In our scheme, Alice will hold $A$ as her share of the key and Bob will hold $B$ as his key share. They are committed to their share by being committed to a poly-size classical description of the operator applied. We sketch why the scheme has the two necessary properties.

1. Since the authentication is performed using only Clifford gates, an approach as in [4] will allow to fairly easily apply Clifford gates "through the authentication". Since the Clifford unitaries form a group, the operation consisting of decrypting a qubit, applying a Clifford gate to it and reauthenticating it using a different key is also Clifford unitary. Hence, we can apply a Clifford gate to a swaddled qubit simply by changing the authentication keys in the appropriate manner. More precisely, to execute the Clifford gate $G \in \mathfrak{C}_{1}$, Alice updates her key to $A\left(G^{\dagger} \otimes \mathbb{1}_{n}\right)$, and Alice and Bob use a TPC to update Alice's commitment to her key. Executing CNOT gates is similar, but involves two swaddlings. The R-gate requires new techniques reminiscent of the fault tolerant implementation of it [1467]. The details appear within, but we basically reduce it to securely producing a state $|0\rangle$, a magic state, a measurement in the computational basis, and applying secure Clifford gates together with a carefully chosen secure classical computation which tells the parties how to update their keys.

2. If Bob is to check an authenticated qubit, we simply give him $|V\rangle$ and he applies $B^{\dagger}$ and measures the first $n$ wires, rejecting if they are not all zero. After that he re-applies $B$ to recover the authentication $|V\rangle$. If Alice is to perform the check, we perform a secure classical computation where the inputs are the committed descriptions of $A$ and $B$ which effectively swaps the inner and outer authentications (see below for details). Alice then holds the outermost authentication and can easily test its integrity.

The final state of the computation is obtained after each party reveals the authentication keys needed by the other party to open its output wires. 


\section{Preliminaries}

The set of linear operators from and to Hilbert space $\mathcal{A}$ is denoted by $\mathrm{L}(\mathcal{A})$. The set of trace 1 positive semi-definite operators is denoted by $\mathrm{D}(\mathcal{A})$; it is the set of quantum states for register $\mathcal{A}$. For $\rho, \rho^{\prime} \in \mathrm{D}(\mathcal{A})$, we denote by $\Delta\left(\rho, \rho^{\prime}\right):=\frac{1}{2}\left\|\rho-\rho^{\prime}\right\|_{1}$ the trace norm distance between $\rho$ and $\rho^{\prime}$. Finally, we denote by $\mathfrak{C}_{n}$ the set of Clifford operators on $n$ qubits. More information can be found in [5].

\subsection{Secure Two-Party Classical Computation against Quantum Adversaries}

Our protocol will use various classical two-party computations throughout its execution, each modeled as an ideal functionality. Recent work [12 11] show that composable classical two-party computation protocols can be devised with security against quantum adversaries provided some classical computational assumptions hold against this class of adversaries. One example of such an assumption is learning with errors is hard [13 11]. The framework in [11] allows us to replace the ideal functionalities with such secure protocols. Here we therefore focus on proving security given the ideal functionalities.

In the following, the ideal functionality for string commitment will be denoted by $\mathrm{id}_{\mathrm{sc}}$. It is defined as follows:

$$
\begin{aligned}
\operatorname{id}_{\mathrm{sc}}((i d, s), \perp) & =(\perp,(i d, \text { committed })) \text { if } i d \text { is new, } \\
\operatorname{id}_{\mathrm{sc}}(\perp,(i d, s)) & =((i d, \text { committed }), \perp) \text { if } i d \text { is new, } \\
\operatorname{id}_{\mathrm{sc}}\left(s, s^{\prime}\right) & =(\perp, \perp) \text { otherwise } .
\end{aligned}
$$

In order for Alice to commit on $s \in\{0,1\}^{*}$, Alice and Bob call id $\mathrm{ic}_{\mathrm{sc}}((i d, s), \perp)$, where $i d$ is an unused identifier chosen by Alice. In order for Bob to commit on $s$, Alice and Bob call $\operatorname{id}_{\mathrm{sc}}(\perp,(i d, s))$, where $i d$ is chosen by Bob. The opening of a commitment is performed by calling the ideal functionality $\mathrm{id}_{\text {OPEN }}$ defined as:

$$
\begin{aligned}
\operatorname{id}_{\text {OPEN }}(i d, i d) & =(\perp, s) \text { if } \mathrm{id}_{\mathrm{sc}}((i d, s), \perp) \text { was performed }, \\
\operatorname{id}_{\text {OPEN }}(i d, i d) & =(s, \perp) \text { if } \operatorname{id}_{\mathrm{sc}}(\perp,(i d, s)) \text { was performed, } \\
\operatorname{id}_{\mathrm{sc}}(\cdot, \cdot) & =(\perp, \perp) \text { otherwise } .
\end{aligned}
$$

Note that state has to be passed from $\operatorname{id}_{\mathrm{sc}}($,$) to \operatorname{id}_{\text {open }}($,$) for the above descriptions$ to make sense. Our framework exactly allows that an ideal functionality from an earlier round passes its state to an ideal functionality in a later round. In the framework of [11] they would be considered one ideal functionality, with a state. We prefer the above notation for brevity of later protocol descriptions.

Ideal functionalities computing functions applied to committed values can now be easily defined. Suppose that Alice and Bob want to let Alice learn a function $f$ : $\{0,1\}^{*} \times\{0,1\}^{*} \rightarrow\{0,1\}^{*}$ upon two committed values, $s$ and $s^{\prime}$, where $s$ is committed upon by Alice under $i d$ and $s^{\prime}$ is committed upon by Bob under $i d^{\prime}$. It suffices to define the ideal functionality $\mathrm{id}_{f}^{*}$ as

$$
\begin{aligned}
\operatorname{id}_{f}^{*}\left(\left(i d, i d^{\prime}\right),\left(i d, i d^{\prime}\right)\right) & =\left(f\left(s, s^{\prime}\right), \perp\right) \text { if } \operatorname{id}_{\mathrm{OPEN}}(i d, i d)=(\perp, s) \wedge \mathrm{id}_{\mathrm{OPEN}}\left(i d^{\prime}, i d^{\prime}\right)=\left(s^{\prime}, \perp\right), \\
\operatorname{id}_{f}^{*}(\cdot, \cdot) & =(\perp, \perp) \text { otherwise . }
\end{aligned}
$$


The same if it is Bob who is to learn the output, but with id ${ }_{f}^{*}\left(\left(i d, i d^{\prime}\right),\left(i d, i d^{\prime}\right)\right)=$ $\left(\perp, f\left(s, s^{\prime}\right)\right)$. The same construction can be used to implement any efficiently computable function $f$ evaluated upon any number of committed values. The ideal functionality can also easily be extended to produce commitments to the outputs of $f$. It is this extended ideal functionality we use most often. Again, $\operatorname{id}_{f}^{*}(\cdot, \cdot)$ will have to share state with $\mathrm{id}_{\mathrm{sc}}(\cdot, \cdot)$ and $\operatorname{id}_{\text {opeN }}(\cdot, \cdot)$, which is allowed in our framework.

Note that all the above ideal functionalities are defined such that at most one party has a non-trivial output (i.e., an output which is not known before the inputs are provided). We avoid using functions where both parties have a non-trivial output as an easy way to deal with the problem that fairness in classical secure two-party computation is provably impossible for most functionalities. It is not hard to see that it follows from known completeness results of quantum-secure classical two-party computation[1211] that the classical ideal functionalities specified above can be implemented with security against poly-time quantum adversaries. We skip the details of this, as our focus here is on using the classical ideal functionalities for constructing secure two-party protocols for computing on quantum data.

\subsection{Clifford-Based Quantum Authentication}

In order to detect misbehaviors of an active adversary, we will be evaluating a circuit upon authenticated quantum bits. We will be using a quantum authentication scheme (QAS) [2] based on Clifford operators introduced in [1] as our main building block.

Definition 2.1 (Quantum authentication scheme). A quantum authentication scheme is a set of encryption and decryption superoperators $\left\{\left(\mathscr{E}_{k} S \rightarrow C, \mathscr{D}_{k}^{C \rightarrow S F}\right): k \in \mathcal{K}\right\}$, where $\mathcal{K}$ is the set of possible keys, $S$ is the input system, $C$ is the ciphertext system, and $F$ is a "flag" system that contains either $|\mathrm{acc}\rangle$ or $|\mathrm{rej}\rangle$. A QAS is such that for all $k \in \mathcal{K},\left(\mathscr{D}_{k} \circ \mathscr{E}_{k}\right)\left(\rho_{S}\right)=\rho_{S} \otimes|\operatorname{acc}\rangle\left\langle\left.\operatorname{acc}\right|_{F}\right.$.

A QAS is secure if it satisfies the following:

Definition 2.2 (Security of a QAS). Let $\mathscr{E}_{k}^{S \rightarrow C}$ and $\mathscr{D}_{k}^{C \rightarrow S F}$ be the encoder and decoder corresponding to key $k$. Then, we say that the $Q A S(\mathscr{E}, \mathscr{D})$ is $\varepsilon$-secure if, for all attacks $U_{C R}$, there exists two $C P$ maps $\mathscr{U}_{R \rightarrow R}^{\text {acc }}$ and $\mathscr{U}_{R \rightarrow R}^{\text {rej }}$ with $\mathscr{U}^{\text {acc }}+\mathscr{U}^{\text {rej }}=\mathbb{1}$ such that for all inputs $\psi_{S R}$, we have that for some fixed state $\Omega_{S}$ :

$$
\begin{aligned}
& \| \frac{1}{\# \mathcal{K}} \sum_{k \in \mathcal{K}} \mathscr{D}_{k}\left(U_{C R} \mathscr{E}_{k}\left(\psi_{S R}\right) U_{C R}^{\dagger}\right) \\
& \quad-\left(\mathscr{U}^{\mathrm{acc}}\left(\psi_{S R}\right) \otimes|\operatorname{acc}\rangle\left\langle\left.\operatorname{acc}\right|_{F}+\mathscr{U}^{\mathrm{rej}}\left(\psi_{R}\right) \otimes \Omega_{S} \otimes \mid \operatorname{rej}\right\rangle\left\langle\left.\operatorname{rej}\right|_{F}\right) \|_{1} \leqslant \varepsilon .\right.
\end{aligned}
$$

This definition can be shown to be equivalent to the existence of a simulator that interacts only with an ideal functionality in which Eve's only choice is whether or not to destroy the state and cause a rejection. 
Definition 2.3 (Clifford-based QAS[1]). Let $S$ be an $s$-qubit system, $A$ be an $n$-qubit system, and let $C=S \otimes A$. Let $\mathcal{K}$ index all Clifford unitaries $C_{k}$ on $s+n$ qubits. Then, the Clifford-based QAS is defined by the following encryption and decryption maps where $P_{\mathrm{acc}}^{S A}=\mathbb{1}_{S} \otimes\left|0^{n}\right\rangle\left\langle\left. 0^{n}\right|_{A}\right.$ and $P_{\mathrm{rej}}^{S A}=\mathbb{1}_{S A}-P_{\mathrm{acc}}^{S A}$ :

$$
\begin{aligned}
\mathscr{E}_{k}\left(\rho_{S}\right) & =C_{k}\left(\rho_{S} \otimes\left|0^{n}\right\rangle\left\langle\left. 0^{n}\right|_{A}\right) C_{k}^{\dagger},\right. \\
\mathscr{D}_{k}\left(\sigma_{S A}\right) & =\operatorname{tr}_{A}\left(P _ { \mathrm { acc } } C _ { k } ^ { \dagger } \sigma _ { S A } C _ { k } P _ { \mathrm { acc } } \otimes | \operatorname { a c c } \rangle \left\langle\left.\operatorname{acc}\right|_{F}\right.\right. \\
& \quad+\operatorname{tr}\left(P_{\mathrm{rej}} C_{k}^{\dagger} \sigma_{S A} C_{k}\right) \pi_{S A} \otimes|\mathrm{rej}\rangle\left\langle\left.\mathrm{rej}\right|_{F}\right),
\end{aligned}
$$

where $\pi_{S A}$ is an arbitrary fixed state that the decoder outputs when it rejects the authentication.

The following establishes the security of the QAS based on random Clifford operators. The proof of security is more or less the same as in [1] :

Theorem 2.4 (Security of Clifford-based QAS). The QAS defined above is $\varepsilon(n)$ secure for $\varepsilon(n)=6 \times 2^{-n}$.

It should be mentioned that picking a random Clifford operation acting upon $\ell$ qubits requires to pick a uniformly random poly $(\ell)$-bit classical key $k$ and the mapping between $k$ and the corresponding Clifford operation can be performed efficiently [6]1]. In other words, the key size of the Clifford-based QAS is polynomial in the number of qubits $\ell=s+n$ used to authenticate an $s$-qubit quantum state.

\subsection{Two-Party Quantum Protocols}

We define two-party strategies in a similar way as in [4]10], with some adaptations made for the fact that we are computing a CPTP map and not just a unitary and that we allow ideal functionalities of different rounds to share states (equivalent to considering one, stateful functionality). Two-party protocols for the evaluation of some CPTP map are particular cases of two-party strategies. Two-party strategies have access to some oracle in each round. An oracle is just a CPTP map acting on registers at both Alice and Bob. Oracles implement some functionalities like a communication channel or some more complex two-party functionalities.

An $m$-turn two-party strategy with oracle calls is defined by tuples of quantum operations $\mathscr{A}:=\left(\mathscr{A}_{1}, \ldots, \mathscr{A}_{m+1}\right), \mathscr{B}:=\left(\mathscr{B}_{1}, \ldots, \mathscr{B}_{m+1}\right)$, and $\mathscr{O}:=\left(\mathscr{O}_{1}, \ldots, \mathscr{O}_{m}\right)$. For $i \in[1 . . m+1]$, operations $\mathscr{A}_{i} \in \mathrm{L}\left(\mathcal{A}_{i-1} \otimes \mathcal{A}_{i-1}^{\mathscr{O} \text {,out }}\right) \mapsto \mathrm{L}\left(\mathcal{A}_{i} \otimes \mathcal{A}_{i}^{\mathscr{O}, \text { in }}\right)$ and $\mathscr{B}_{i} \in$ $\mathrm{L}\left(\mathcal{B}_{i-1} \otimes \mathcal{B}_{i-1}^{\mathscr{O}, \text { out }}\right) \mapsto \mathrm{L}\left(\mathcal{B}_{i} \otimes \mathcal{B}_{i}^{\mathscr{O}, \text { in }}\right)$ are the actions performed at turn $i$ by Alice and Bob respectively. The operation $\mathscr{O}_{i}: \mathrm{L}\left(\mathcal{A}_{i}^{\mathscr{O}, \text { in }} \otimes \mathcal{O}_{i-1} \otimes \mathcal{B}_{i}^{\mathscr{O}, \text { in }}\right) \mapsto \mathrm{L}\left(\mathcal{A}_{i}^{\mathscr{O} \text {, out }} \otimes \mathcal{O}_{i} \otimes \mathcal{B}_{i}^{\mathscr{O} \text {, out }}\right)$ models the oracle provided to Alice and Bob at turn $i$, and $\mathcal{O}_{i}$ a register used for passing state from the oracle of one round to the oracle at the next round. The oracle at turn $i \in[1 . . m]$ takes place right after $\mathscr{A}_{i}$ and $\mathscr{B}_{i}$ have been applied. In particular, these operations set the input registers $\mathcal{A}_{i-1}^{\mathscr{O} \text {,in }}$ and $\mathcal{B}_{i-1}^{\mathscr{O} \text {,in }}$ for the call to $\mathscr{O}_{i}$. The outputs are available to Alice and Bob in the next turn, in the output registers $\mathcal{A}_{i}^{\mathscr{O} \text {,out }}$ and $\mathcal{B}_{i}^{\mathscr{O} \text {,out }}$. We make 
one exception to this general form, the last turn (that is turn $m+1$ ) of a strategy does not invoke any oracle, and is there simply to allow Alice and Bob to post-process the output of the last oracle.

Let $\Pi=(\mathscr{A}, \mathscr{B}, \mathscr{O}, m)$ be an $m$-turn two-party strategy with oracle calls. The final state of the interaction between $\mathscr{A}$ and $\mathscr{B}$ upon joint input state $\rho_{\text {in }} \in \mathrm{D}\left(\mathcal{A}_{0} \otimes \mathcal{B}_{0} \otimes \mathcal{R}\right)$, where $\mathcal{R}$ is a reference system with $\operatorname{dim} \mathcal{R}=\operatorname{dim} \mathcal{A}_{0} \operatorname{dim} \mathcal{B}_{0}$, is denoted by

$$
\begin{aligned}
{[\mathscr{A} \circledast \mathscr{B}]^{\mathscr{O}}\left(\rho_{\text {in }}\right):=} & \left(\mathscr{A}_{m+1} \otimes \mathscr{B}_{m+1} \otimes \mathbb{1}_{\mathrm{L}\left(\mathcal{R} \otimes \mathcal{O}_{m+1}\right)}\right) \\
& \left(\mathbb{1}_{\mathrm{L}\left(\mathcal{A}_{m} \otimes \mathcal{B}_{m} \otimes \mathcal{R}\right)} \otimes \mathscr{O}_{m}\right)\left(\mathscr{A}_{m} \otimes \mathscr{B}_{m} \otimes \mathbb{1}_{\mathrm{L}\left(\mathcal{R} \otimes \mathcal{O}_{m}\right)}\right) \\
& \ldots\left(\mathbb{1}_{\mathrm{L}\left(\mathcal{A}_{1} \otimes \mathcal{B}_{1} \otimes \mathcal{R}\right)} \otimes \mathscr{O}_{1}\right)\left(\mathscr{A}_{1} \otimes \mathscr{B}_{1} \otimes \mathbb{1}_{\mathrm{L}(\mathcal{R})}\right)\left(\rho_{\mathrm{in}}\right) .
\end{aligned}
$$

A communication oracle from Alice to Bob is modeled by having $\mathcal{A}_{i}^{\mathscr{O}} \approx \mathcal{B}_{i}^{\mathscr{O}}$ and letting $\mathscr{O}_{i}$ move the state in $\mathcal{A}_{i}^{\mathscr{O}}$ to $\mathcal{B}_{i}^{\mathscr{O}}$. A classical ideal functionality, as those described in Sect. 2.1, can easily be made available as oracle calls. We assume that in each round $i$ at most one classical ideal functionality is applied. The parties place their inputs in the appropriate registers $\mathcal{A}_{i-1}^{\mathscr{O}}$ and $\mathcal{B}_{i-1}^{\mathscr{O}}$. The operation of the oracle $\mathscr{O}_{i}$ is as follows: it measures the input registers $\mathcal{A}_{i-1}^{\mathscr{O}}$ and $\mathcal{B}_{i-1}^{\mathscr{O}}$ to force classical inputs. Then, it applies the appropriate classical ideal functionality on those classical inputs plus its classical internal state found in $\mathcal{O}_{i-1}$. This produces outputs for the parties and a new internal state. The outputs are placed in $\mathcal{A}_{i}^{\mathscr{O}}$ and $\mathcal{B}_{i}^{\mathscr{O}}$. The new state is placed in $\mathcal{O}_{i}$.

A two-party hybrid protocol for $\mathscr{F}: \mathrm{L}\left(\mathcal{A}_{\text {in }} \otimes \mathcal{B}_{\text {in }}\right) \rightarrow \mathrm{L}\left(\mathcal{A}_{\text {out }} \otimes \mathcal{B}_{\text {out }}\right)$ between parties $\mathscr{A}$ and $\mathscr{B}$ upon joint input state $\rho_{\text {in }} \in \mathrm{D}\left(\mathcal{A}_{\text {in }} \otimes \mathcal{B}_{\text {in }} \otimes \mathcal{R}\right)$ is defined as:

Definition 2.5. An $m$-turn two-party hybrid protocol $\Pi_{\mathscr{F}}^{\mathscr{O}}=(\mathscr{A}, \mathscr{B}, \mathscr{O}, m)$ for $\mathscr{F}$ : $\mathrm{L}\left(\mathcal{A}_{\text {in }} \otimes \mathcal{B}_{\text {in }}\right) \rightarrow \mathrm{L}\left(\mathcal{A}_{\text {out }} \otimes \mathcal{B}_{\text {out }}\right)$ is a $m$-turn two-party strategy with oracle calls, where $\mathcal{A}_{0}:=\mathcal{A}_{\text {in }}, \mathcal{B}_{0}:=\mathcal{B}_{\text {in }}, \mathcal{A}_{m+1}:=\mathcal{A}_{\text {out }}, \mathcal{B}_{m+1}:=\mathcal{B}_{\text {out }}$, and where for all $\rho_{\text {in }} \in \mathrm{D}\left(\mathcal{A}_{0} \otimes \mathcal{B}_{0} \otimes \mathcal{R}\right), \Delta\left([\mathscr{A} \circledast \mathscr{B}]^{\mathscr{O}}\left(\rho_{\text {in }}\right),\left(\mathscr{F} \otimes \mathbb{1}_{\mathcal{R}}\right)\left(\rho_{\text {in }}\right)\right)=0$. In the following, we often write simply two-party protocol to refer to a two-party hybrid protocol.

For $i \in[0 . . m]$, the joint state after turn $i+1$ in $\Pi_{\mathscr{F}}^{\mathscr{O}}$ is denoted by $[\mathscr{A} \circledast \mathscr{B}]_{i+1}^{\mathscr{O}}\left(\rho_{\text {in }}\right):=$ $\left(\mathbb{1}_{\mathrm{L}\left(\mathcal{B}_{i+1} \otimes \mathcal{A}_{i+1} \otimes \mathcal{R}\right)} \otimes \mathscr{O}_{i+1}\right)\left(\mathscr{A}_{i+1} \otimes \mathscr{B}_{i+1} \otimes \mathbb{1}_{\mathrm{L}\left(\mathcal{R} \otimes \mathcal{O}_{i+1}\right)}\right)[\mathscr{A} \circledast \mathscr{B}]_{i}^{\mathscr{O}}\left(\rho_{\text {in }}\right)$, where $[\mathscr{A} \circledast$ $\mathscr{B}]_{0}^{\mathscr{O}}\left(\rho_{\mathrm{in}}\right):=\rho_{\mathrm{in}}$, and $[\mathscr{A} \circledast \mathscr{B}]_{m+1}^{\mathscr{O}}\left(\rho_{\text {in }}\right):=[\mathscr{A} \circledast \mathscr{B}]^{\mathscr{O}}\left(\rho_{\text {in }}\right)$.

\section{Modeling Active Security}

We start by extending the framework in [4] to handle active security. Our model is very standard, defining security via simulation, but for completeness we describe and motivate the changes made in [5].

Let $\Pi_{\mathscr{F}}^{\mathscr{O}}=(\mathscr{A}, \mathscr{B}, \mathscr{O}, m)$ be a $m$-turn two-party hybrid protocol. Let $\tilde{\mathscr{A}}$ and $\tilde{\mathscr{B}}$ be adversaries in $\Pi_{\mathscr{F}}^{\mathscr{O}}$. We denote by $[\tilde{\mathscr{A}} \circledast \mathscr{B}]^{\mathscr{O}}$ and $[\mathscr{A} \circledast \tilde{\mathscr{B}}]^{\mathscr{O}}$ the resulting $m$-turn twoparty strategies. We will as usual define the security of such actively attacked protocols by comparing to a simulation. The simulation is basically an ideally secure evaluation of $\mathscr{F}$.

The ideally secure protocol for evaluating $\mathscr{F}$ would be in a world where $\mathscr{F}$ actually existed as an oracle - the parties would simply call this oracle. We can, however, not expect any protocol to be as secure as this, as for most protocols we cannot ensure that 
either both parties get the output or no party gets the output, known as fairness, which is ensured in the above ideal setting. We will therefore consider a setting where one of the parties learns its output first, and where the party learning it first, if corrupted, can prevent the other party from learning its output. We will only give the definition for the case where Alice learns first. Deriving the definition for the symmetric case where Bob learns first is trivial.

To avoid confusion between the parties in the real protocol and in the ideal protocol, we formulate the ideal protocol with parties Charleen, $\mathcal{C}$, and Dan, $\mathcal{D}$, taking the seats of Alice respectively Bob. So, we look at an ideal functionality $\mathscr{F}: \mathrm{L}\left(\mathcal{C}_{\text {in }} \otimes \mathcal{D}_{\text {in }}\right) \rightarrow$ $\mathrm{L}\left(\mathcal{C}_{\text {out }} \otimes \mathcal{D}_{\text {out }}\right)$, but where we keep a mental note reminding that $\mathcal{C}_{\text {in }}:=\mathcal{A}_{\text {in }}, \mathcal{C}_{\text {out }}:=$ $\mathcal{A}_{\text {out }}, \mathcal{D}_{\text {in }}:=\mathcal{B}_{\text {in }}$ and $\mathcal{D}_{\text {out }}:=\mathcal{B}_{\text {out }}$.

The ideal protocol for $\mathscr{F}$ is then a 2-turn two-party hybrid protocol $\Gamma_{\mathscr{F}}=$ $(\mathscr{C}, \mathscr{D}, \mathscr{F}, 2)$, which lets the parties query $\mathscr{F}$ in turn 1 , but only letting $\mathcal{C}$ see her output in turn 1 . In turn 2 , Charleen then inputs a bit $f$, with $f=1$ indicating that Dan should receive his output and $f=0$ indicating that Dan should not receive his output. Dan will receive $f$, and if $f=1$ he will additionally be given his output. This is handled by the second oracle. Then the parties output whatever they received from the oracles. In the honest protocol, Charleen always inputs $f=1$. We call this the ideal protocol for evaluating $\mathscr{F}$ without fairness for Dan.

The ideal protocol $\Gamma_{\mathscr{F}}=(\mathscr{C}, \mathscr{D}, \mathscr{F}, 2)$ for $\mathscr{F}$ without fairness for Dan:

1. By convention we have $\mathcal{C}_{0}=\mathcal{C}_{\text {in }}$ and $\mathcal{D}_{0}=\mathcal{D}_{\text {in }}$ and that $\mathcal{O}_{0}$ is empty. In the ideal protocol we let $\mathcal{C}_{1}^{\mathscr{O} \text {,in }}=\mathcal{C}_{0}$ and $\mathcal{D}_{1}^{\mathscr{F} \text {,in }}=\mathcal{D}_{0}$, we let $\mathcal{C}_{1}$ and $\mathcal{D}_{1}$ be empty, and $\mathscr{C}_{1}=$ $\mathbb{1}_{\mathrm{L}\left(\mathcal{C}_{0}\right)}$ and $\mathscr{D}_{1}=\mathbb{1}_{\mathrm{L}\left(\mathcal{D}_{0}\right)}$. I.e., in turn 1 the parties simply send their inputs to the first oracle $\mathscr{F}_{1}$. For the first oracle $\mathscr{F}_{1}: \mathrm{L}\left(\mathcal{C}_{1}^{\mathscr{F}, \text { in }} \otimes \mathcal{O}_{0} \otimes \mathcal{D}_{1}^{\mathscr{F}, \text { in }}\right) \mapsto \mathrm{L}\left(\mathcal{C}_{1}^{\mathscr{F}, \text { out }} \otimes\right.$ $\mathcal{O}_{1} \otimes \mathcal{D}_{1}^{\mathscr{F} \text {,out }}$, we set $\mathcal{C}_{1}^{\mathscr{F} \text {,out }} \approx \mathcal{C}_{\text {out }}, \mathcal{O}_{1} \approx \mathcal{D}_{\text {out }}$ and we let $\mathcal{D}_{1}^{\mathscr{F} \text {,out }}$ be empty. We let $\mathscr{F}_{1}=\mathscr{F}$. I.e., $\mathscr{F}_{1}$ simply applies $\mathscr{F}$ to the inputs supplied by the parties, sends

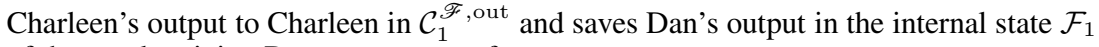
of the oracle, giving Dan no output so far.

2. We let $\mathcal{C}_{2} \approx \mathcal{C}_{1}^{\mathscr{F} \text {,out }}\left(\approx \mathcal{C}_{\text {out }}\right)$ and we let $\mathcal{C}_{2}^{\mathscr{F}}$,in be a one qubit register, holding a qubit we name $|f\rangle$. We let $\mathscr{C}_{2}=\mathbb{1}_{\mathrm{L}\left(\mathcal{C}_{1}^{\mathscr{F}}, \text { out }\right.}{ }_{\left.\mathcal{C}_{2}\right)} \otimes|1\rangle$, i.e., it moves the output from the oracle

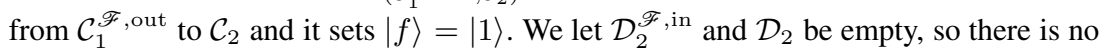
need to specify $\mathscr{D}_{2}$.

For the second oracle $\mathscr{O}_{2}: \mathrm{L}\left(\mathcal{C}_{2}^{\mathscr{F} \text {,in }} \otimes \mathcal{O}_{1} \otimes \mathcal{D}_{2}^{\mathscr{F}, \text { in }}\right) \mapsto \mathrm{L}\left(\mathcal{C}_{2}^{\mathscr{F}, \text { out }} \otimes \mathcal{O}_{2} \otimes \mathcal{D}_{2}^{\mathscr{F} \text {, out }}\right)$, we let $\mathcal{C}_{2}^{\mathscr{F} \text {,out }}$ and $\mathcal{O}_{2}$ be empty, and we let $\mathcal{D}_{2}^{\mathscr{F}}$,out be of the same dimension as $\mathcal{D}_{\text {out }}$, plus room for one qubit $|a\rangle$. It starts by measuring $|f\rangle$ in the computational basis. If $|f\rangle=|1\rangle$, it then sets $\mathcal{D}_{2}^{\mathscr{F}}$, out to hold $|a\rangle=|1\rangle$ along with the state in $\mathcal{O}_{1}$. If $|f\rangle=|0\rangle$, it sets $\mathcal{D}_{2}^{\mathscr{F} \text {,out }}$ to hold $|a\rangle=|0\rangle$ along with some fixed dummy state $|\perp\rangle$ of the right dimension to fill $\mathcal{C}_{2}^{\mathscr{F} \text {,out }}$. I.e., if Charleen inputs $f=1$, then Dan will get his output. If $f=0$, Dan gets no output, except a bit $a=0$ telling him that Charleen cheated him of his output (we say that Charleen aborted the computation).

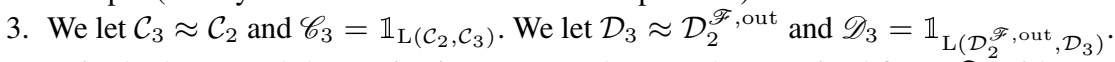
I.e., in the last round the parties just output whatever they received from $\mathscr{F}$, with Dan possibly outputting a dummy state, in case of abort.

Consider the powers of a corrupted Charleen, $\tilde{\mathscr{C}}$. She might in the first round provide an alternative input for $\mathscr{F}$, possibly saving her original input, or a part thereof, in some 
ancilla. This is input substitution. Her choice of alternative input can only depend on her own original input, not that of Dan. This is input independence. Then she learns only the output of the oracle. This is privacy. After learning her own output she might then specify that Dan is not to learn his output. This is the necessary lack of fairness. A corrupted Dan has similar powers, except that he cannot abort after seeing his output. We then say that a protocol is secure if it only allows attacks which could be mounted in the ideal protocol, i.e., it only allows the inevitable attack.

Definition 3.1. Let $\Pi_{\mathscr{F}}^{\mathscr{O}}=(\mathscr{A}, \mathscr{B}, \mathscr{O}, m)$ be a two-party hybrid protocol for $\mathscr{F}$ : $\mathrm{L}\left(\mathcal{A}_{\text {in }} \otimes \mathcal{B}_{\text {in }}\right) \rightarrow \mathrm{L}\left(\mathcal{A}_{\text {out }} \otimes \mathcal{B}_{\text {out }}\right)$. Let $\Gamma_{\mathscr{F}}$ be the ideal protocol for $\mathscr{F}$ without fairness for Dan. Let $\delta \in[0 . .1]$. We say that $\Pi_{\mathscr{F}}^{\mathscr{O}}$ is $\delta$-active secure without fairness for Bob, iffor all adversaries $\tilde{\mathscr{A}}$ and $\tilde{\mathscr{B}}$ in $\Pi_{\mathscr{F}}^{\mathscr{O}}$, there exist adversaries $\tilde{\mathscr{C}}$ and $\tilde{\mathscr{D}}$ in $\Gamma_{\mathscr{F}}$ with sizes polynomial in the sizes of $\tilde{\mathscr{A}}$ and $\tilde{\mathscr{B}}$ such that for all input states $\rho_{\mathrm{in}} \in \mathrm{D}\left(\mathcal{A}_{\text {in }} \otimes \mathcal{B}_{\text {in }} \otimes \mathcal{R}\right)$,

$$
\Delta\left([\tilde{\mathscr{A}} \circledast \mathscr{B}]^{\mathscr{O}}\left(\rho_{\text {in }}\right),[\tilde{\mathscr{C}} \circledast \mathscr{D}]^{\mathscr{F}}\left(\rho_{\text {in }}\right)\right) \leq \delta \& \Delta\left([\mathscr{A} \circledast \tilde{\mathscr{B}}]^{\mathscr{O}}\left(\rho_{\text {in }}\right),[\mathscr{C} \circledast \tilde{\mathscr{D}}]^{\mathscr{F}}\left(\rho_{\text {in }}\right)\right) \leq \delta
$$

If a protocol is $\delta$-active secure for $\delta=0$, then it is called perfectly active secure. If it is $\delta$-active secure for $\delta$ negligible in some security parameter $n$, then it is called statistically active secure (in $n$ ).

\section{Securely Swaddling Wires to Ensure Ultimate Speciousness}

The Clifford based QAS, described in Sect.2.2, can be used to share the authentication of a quantum message in a way that allows any of the players, when helped by a TPC, to verify that a state has not been tampered with, and this without being able to get information on the encoded state. This primitive will be used extensively in our protocol to ensure that all players are ultimately specious. It relies on a string commitment scheme and secure TPCs provided as oracles.

The basic idea consists in swaddling each input wire w into a set of $2 n$ dummy wires where $n$ belongs to Alice and $n$ belongs to Bob. No party will be able to extract information about the state of the original wire from the swaddling. Moreover, any attempt to modify the state of the original wire will be detected except with negligible probability in $n$. The subprotocol Swaddle(w), described below, uses two application of the Clifford-based QAS (one on top of the other) in order to achieve this. Alice authenticates the state of her wire $\mathrm{w}$ and commits to her authentication key $K_{\mathrm{w}, a}$ using identifier $i d_{A}(\mathrm{w})$. She then sends the resulting system to Bob who authenticates it using key $K_{\mathrm{w}, b}$ that he also commits upon using identifier $i d_{B}(\mathrm{w})$. Bob sends back the resulting system to Alice allowing her to test the validity of the swaddling. The swaddling therefore uses a total of $2 n+1$ wires, where Alice holds the innermost and Bob the outermost key of the resulting swaddling $\mathfrak{s}(\mathrm{w})$ of wire $\mathrm{w}$.

Consider now the test performed by Alice at Step 7 Let $A$ and $B$ be the Clifford operators corresponding to keys $K_{\mathrm{w}, a}$ and $K_{\mathrm{w}, b}$ respectively. Notice that Alice can easily test the authenticity of a swaddling when she holds the outermost authentication. She simply applies $A^{\dagger}$ upon the $2 n+1$ wires, measures her $n$ dummy wires in the computational basis to verify that they are in state $\left|0^{n}\right\rangle$. She then re-applies $A$ to the $2 n+1$ 
wires of the swaddling. If Alice holds the innermost authentication of the swaddling, the testing procedure relies on the ideal functionality $\mathrm{id}_{\mathrm{TEST}}$ defined as:

$\operatorname{id}_{\text {тЕST }}\left(\left(i_{a}, i_{b}\right),\left(i_{a}^{\prime}, i_{b}^{\prime}\right)\right)= \begin{cases}\left(\left(t, K_{\mathrm{w}, a}^{\prime}\right), K_{\mathrm{w}, b}^{\prime}\right) & \text { if } i_{a}=i_{a}^{\prime}=i d_{A}(\mathrm{w}) \wedge i_{b}=i_{b}^{\prime}=i d_{B}(\mathrm{w}), \\ (\perp, \perp) & \text { otherwise, }\end{cases}$

where $K_{\mathrm{w}, a}^{\prime}$, and $K_{\mathrm{w}, b}^{\prime}$ correspond to random Clifford operations $A^{\prime} \in \mathfrak{C}_{2 n+1}$ and $B^{\prime} \in$ $\mathfrak{C}_{n+1}$ respectively, and $t$ corresponds to Clifford $T \in \mathfrak{C}_{2 n+1}$ subject to:

1. $\operatorname{id}_{\text {OPEN }}\left(i d_{A}(\mathrm{w}), i d_{A}(\mathrm{w})\right)=\left(\perp, K_{\mathrm{w}, a}^{\prime}\right)$ and $\operatorname{id}_{\text {oPEN }}\left(i d_{B}(\mathrm{w}), i d_{B}(\mathrm{w})\right)=\left(K_{\mathrm{w}, b}^{\prime}, \perp\right)$, and

2. If Alice holds the innermost key of w then $T=\left(\mathbb{1}_{n} \otimes B^{\prime}\right)\left(A^{\dagger} \otimes \mathbb{1}_{n}\right) B^{\dagger}$.

Swaddle $(\mathcal{W})$, with $\mathcal{W} \subseteq \mathcal{A}:$

1. Alice initializes $n \cdot \overline{\#} \mathcal{W}$ dummy wires in state $|0\rangle$.

2. For each w $\in \mathcal{W}$, Alice randomly chooses Clifford $K_{\mathrm{w}, a}$ on $n+1$ qubits, commits to it in $\operatorname{id}_{\mathrm{sc}}\left(\left(i d_{A}(\mathrm{w}), K_{\mathrm{w}, a}\right), \perp\right)$, and applies it to w and her dummies.

3. Alice sends these $\# \mathcal{W} \cdot(n+1)$ wires to Bob.

4. Bob initializes $\# \mathcal{W} \cdot n$ dummy wires in the state $|0\rangle$.

5. For each $\mathrm{w} \in \mathcal{W}$, Bob randomly chooses a Clifford $K_{\mathrm{w}, b}$ on $2 n+1$ qubits, commits to it in $\operatorname{id}_{\mathrm{sc}}\left(\perp,\left(i d_{B}(\mathrm{w}), K_{\mathrm{w}, b}\right)\right)$, and applies it to the $n+1$ wires received from Alice as well as his own dummies.

6. Bob sends all $\# \mathcal{W} \cdot(2 n+1)$ wires back to Alice. Let $\mathfrak{s}(\mathrm{w})$ denote the resulting swaddling of $\mathrm{w} \in \mathcal{W}$.

7. For each $\mathrm{w} \in \mathcal{W}$, Alice calls TestSwaddling $(\mathfrak{s}(\mathrm{w}))$.

Condition 1 ensures that the authentication keys with identifiers $i d_{A}(\mathrm{w})$ and $i d_{B}(\mathrm{w})$ have been updated to hold values $K_{\mathrm{w}, a}^{\prime}$ and $K_{\mathrm{w}, b}^{\prime}$ respectively. Conditions 2 makes sure that the state $|\sigma\rangle$ of a valid swaddling $\mathfrak{s}(\mathrm{w})$ satisfies $T|\sigma\rangle=\left|0^{n}\right\rangle \otimes B^{\prime}\left(|\varphi\rangle \otimes\left|0^{n}\right\rangle\right)$, where $|\varphi\rangle$ is the logical state of $\mathfrak{s}(\mathrm{w})$. Notice that Alice gets no information about Bob's Clifford $B^{\prime}$ if she had no information about $B$ to start with.

TestSwaddling $(\mathfrak{s}(\mathrm{w}))$, with Alice doing the testing:

1. If Alice holds the outermost authentication key $K_{\mathrm{w}, a}$ then:

- Alice applies $A^{\dagger}$ on $\mathfrak{s}(\mathrm{w})$, where $A$ is the Clifford corresponding to string $K_{\mathrm{w}, a}$,

- Alice tests that her dummies are together in state $\left|0^{n}\right\rangle$. If not then she aborts.

- Alice re-applies $A$ on the $2 n+1$ qubits of the swaddling.

2. Else they call $\left(\left(t, K_{\mathrm{w}, a}^{\prime}\right), K_{\mathrm{w}, b}^{\prime}\right)=\mathrm{id}_{\mathrm{TEST}}\left(\left(i d_{A}(\mathrm{w}), i d_{B}(\mathrm{w})\right),\left(i d_{A}(\mathrm{w}), i d_{B}(\mathrm{w})\right)\right)$,

- If one party gets $\perp$ in id $\mathrm{ieST}_{\mathrm{TEST}}$ then abort.

- Alice applies $T$ on $\mathfrak{s}(\mathrm{w})$ where $T$ is the Clifford operator corresponding to string $t$.

- Alice tests that her dummies are together in state $\left|0^{n}\right\rangle$. If not then she aborts.

- Alice applies $A^{\prime}$ to the the $2 n+1$ qubits of the swaddling (note that $A^{\prime}$ and $B^{\prime}$ are committed upon with identifiers $i d_{A}(\mathrm{w})$ and $i d_{B}(\mathrm{w})$ respectively).

At the end of TestSwaddling $(\mathfrak{s}(\mathrm{w}))$, Alice holds the outermost authentication key $K_{\mathrm{w}, a}^{\prime}$ of the resulting swaddling. Bob can do the testing by the exact same procedure provided the roles of Alice and Bob are reversed in TestSwaddling $(\mathfrak{s}(\mathrm{w}))$. The security of these procedures will be discussed in Sect. 5.5. Intuitively, we expect that TestSwaddling $(\mathfrak{s}(\mathrm{w}))$ allows parties to test that a swaddling $\mathfrak{s}(\mathrm{w})$ has not been tampered with. It will also be used to test that each party transforms a swaddling $\mathfrak{s}(\mathrm{w})$ the 
way they should during the execution of the protocol. Notice that no information about the logical state of wire w leaks to any party in Swaddle(w) and TestSwaddling $(\mathfrak{s}(\mathrm{w}))$ since statistically secure authentication must also encrypt $\mathrm{w}$ [2].

At the end of the our protocol, each party will be asked to verify that the other party's registers upon which the circuit is evaluated are all in the states they should be. This subprotocol is called TestAllSwaddlings. It simply consists in the execution of TestSwaddling $(\mathfrak{s}(\mathrm{w}))$ for all wires w held by each party and its description can be found in [5].

The following subprotocol implements the obvious way the openings of all committed Clifford operators, thereby allowing Alice and Bob to get the final state of the computation. Notice that since Alice learns Bob's secret keys before she unveils her own, our protocol will lack fairness for Bob.

OpenAllSwaddlings:

1. For each wire $w \in \mathcal{A}_{\text {out }}$, Bob reveals to Alice his committed secret key encrypting w, with identifier $i d_{B}(\mathrm{w})$, by calling $\operatorname{id}_{\mathrm{OPEN}}\left(i d_{B}(\mathrm{w}), i d_{B}(\mathrm{w})\right)$.

2. For each wire $w \in \mathcal{B}_{\text {out }}$, Alice reveals to Bob her committed secret key encrypting w, with identifier $i d_{A}(\mathrm{w})$, by calling $\operatorname{id}_{\text {OPEN }}\left(i d_{A}(\mathrm{w}), i d_{A}(\mathrm{w})\right)$.

\section{Description of the Protocol}

We first start by defining the various spaces on which (the honest) Alice and Bob will be working. The circuit that they want to execute acts on some wires in Alice's possession and some in Bob's possession, and these will be swaddled as described above. We will denote by $\mathcal{A}_{u}, \mathcal{B}_{u}$ the spaces corresponding to Alice and Bob's unswaddled wires, reserving $\mathcal{A}$ and $\mathcal{B}$ for the actual swaddled wires.

We first initialize all the qubits by swaddling them, we then perform each gate from the circuit one after the other on the authenticated data. Hence, we need to give subprotocols for the initialization as well as for each of the gates in our universal set.

For all Clifford gates (i.e., all gates in $\mathcal{U G}$ except for $\mathrm{R}$ ), the subprotocols are fairly simple: we use classical two-party computation to reveal a Clifford operation that executes the gate while updating the encryption key; the revealed Clifford then looks uniformly distributed and independent of everything else.

Implementing the R-gate is more involved. We use ideas from fault-tolerant computation, where this gate is implemented by doing gate teleportation via a so-called magic state: one prepares a special state (namely $|M\rangle=\frac{1}{\sqrt{2}}\left(|0\rangle+e^{i \pi / 4}|1\rangle\right)$ ) and then use a teleportation-like circuit, which itself requires only Clifford gates and measurements, to execute the gate. The problem is then reduced to that of producing this magic state, which can be done by a distillation process. The distillation process that we use is exactly the one considered in [3] (where $|M\rangle$ is an " $H$-type magic state" in their language); a description of it can also be found in [5].

\subsection{Main Protocol}

We now give the full description of our protocol, denoted $\widehat{\Pi}_{\mathscr{F}}^{\mathscr{O}^{\prime}}=\left(\mathscr{A}, \mathscr{B}, \mathscr{O}^{\prime}, m\right)$, allowing to evaluate the CPTP map $\mathscr{F}: \mathrm{L}\left(\mathcal{A}_{\text {in }} \otimes \mathcal{B}_{\text {in }}\right) \mapsto \mathrm{L}\left(\mathcal{A}_{\text {out }} \otimes \mathcal{B}_{\text {out }}\right)$ upon joint 
input state $\rho_{\text {in }} \in \mathrm{D}\left(\mathcal{R} \otimes \mathcal{A}_{\text {in }} \otimes \mathcal{B}_{\text {in }}\right)$. The oracle list needed to run the protocol in the hybrid model is provided implicitly in Sect. 5. The operations performed at each step by $\mathscr{A}$ and $\mathscr{B}$ are described informally as the instructions of Alice and Bob respectively. We will view $\mathscr{F}$ as being implemented by a quantum circuit acting on $\mathcal{A}_{\text {in }} \otimes \mathcal{B}_{\text {in }}$ together with ancillas $\mathcal{A}_{a}$ and $\mathcal{B}_{a}$ initialized in state $|0\rangle$ (we shall explain below how to test that ancillas are really in state $|0\rangle$ ). At the end of the circuit, some of these wires become part of the outputs $\mathcal{A}_{\text {out }}$ and $\mathcal{B}_{\text {out }}$, and the rest are part of the environment that will remain encrypted $\left(\mathcal{A}_{e}\right.$ and $\left.\mathcal{B}_{e}\right)$. Let $G_{1}, G_{2}, \ldots, G_{\ell(n)}$ be an enumeration of all gates of the circuit for $\mathscr{F}$ where $\ell(n)$ is polynomial in $n$, and $G_{i}$ is executed before $G_{i+1}$. This protocol calls a number of subprotocols partially described next and in details in [5].

Protocol $\widehat{\Pi}_{\mathscr{F}}^{\mathscr{O}^{\prime}}$ for the evaluation of $\mathscr{F}$ upon joint input $\rho_{\text {in }} \in \mathrm{D}\left(\mathcal{R} \otimes \mathcal{A}_{\text {in }} \otimes \mathcal{B}_{\text {in }}\right):$

1. Alice and Bob run Initialization,

2. For $i=1 \ldots \ell(n)$ :

- If $G_{i}$ is a one-bit Clifford gate applied to wire w then Alice and Bob call OneQubitClifford $\left(G_{i}, \mathfrak{s}(\right.$ w $\left.)\right)$,

- If $G_{i}$ is a CNOT-gate applied to control wire $\mathrm{w}_{c}$ and $\mathrm{w}_{t}$ then Alice and Bob call $\operatorname{CNOT}\left(\mathfrak{s}\left(\mathrm{w}_{c}\right), \mathfrak{s}\left(\mathrm{w}_{t}\right)\right)$,

- If $G_{i}$ is an R-gate applied to wire w then Alice and Bob call RGate( $(\mathfrak{s}(\mathrm{w}))$,

3. Alice and Bob call TestAllSwaddlings,

4. Alice and Bob call OpenAllSwaddlings,

5. Alice and Bob decrypt the swaddlings for all wires in $\mathcal{A}_{\text {out }}$ and $\mathcal{B}_{\text {out }}$ using the keys received by the other party together with their own.

\subsection{Subprotocols}

The Initialization subprotocol prepares all swaddlings required during the evaluation of the circuit. In addition to swaddling all wires holding the qubits upon which the circuit acts, some ancillary states also have to be swaddled: one wire in the magic state and one wire in state $|0\rangle$ per R-gate in the circuit.

Initialization:

1. For each R-gate being applied to a wire in $\mathcal{A}$, Alice adds one additional wire $\mathrm{m}_{a, i}$ initialized in state $|M\rangle=\frac{1}{\sqrt{2}}\left(|0\rangle+e^{i \pi / 4}|1\rangle\right)$ to $\mathcal{A}$.

2. For each R-gate being applied to a wire in $\mathcal{B}$, Bob adds an additional wire $\mathrm{m}_{b, i}$ initialized in state $|M\rangle$ to $\mathcal{B}$.

3. For each R-gate being applied to a wire in $\mathcal{B}$, Alice adds an additional wire $c_{a, i}$ initialized in state $|0\rangle$ to $\mathcal{A}$.

4. For each R-gate being applied to a wire in $\mathcal{A}$, Bob adds an additional wire $c_{b, i}$ initialized in state $|0\rangle$ to $\mathcal{B}$.

5. Alice calls $\operatorname{Swaddle}(\mathcal{A})$.

6. Bob calls Swaddle $(\mathcal{B})$.

7. For every wire $\mathrm{a} \in \mathcal{A}_{a}$ or added in step 3 above, Alice calls VerifyAncilla(s(a)).

8. For every wire $a \in \mathcal{B}_{a}$ or added in step 4 above, Bob calls VerifyAncilla $(\mathfrak{s}(\mathrm{a}))$.

9. For each magic wire $\mathrm{m} \in \mathcal{A}$, Alice calls DistillMagic $(\mathfrak{s}(\mathrm{m}))$.

10. For each magic wire $\mathrm{m} \in \mathcal{B}$, Bob calls DistillMagic $(\mathfrak{s}(\mathrm{m}))$.

Subprotocols VerifyAncilla $(\mathfrak{s}(\mathrm{a}))$ and DistillMagic $(\mathfrak{s}(\mathrm{m}))$ are described in [5]. The following subprotocols evaluates any one-qubit Clifford, the CNOT-gate, respectively the R. The idea behind the one-qubit protocol is to use TPC to compute the gate 
through the authentication. Of course, the TPC needed depends upon the gate. The idea in the CNOT-subprotocol is to take the two swaddled qubits involved and turn them into one big swaddling. Then, performing a CNOT on them is no different from performing any other Clifford gate on the whole block. We then separate the big swaddling back into its components.

OneQubitClifford(C, $\mathfrak{s}(\mathrm{w}))$ with $\mathrm{w} \in \mathcal{A}$ :

1. Alice and Bob perform a TPC whose outcome tells Alice a randomly chosen Clifford gate which performs the gate, and changes the QAS key.

2. Alice performs the gate on $\mathfrak{s}(w)$.

$\operatorname{CNOT}\left(\mathfrak{s}\left(\mathrm{w}_{\mathrm{c}}\right), \mathfrak{s}\left(\mathrm{w}_{\mathrm{t}}\right)\right)$ with wire $\mathrm{w}_{c} \in \mathcal{A}$ as control and $\mathrm{w}_{t}$ as target:

1. Bob sends $\mathfrak{s}\left(\mathrm{w}_{t}\right)$ to Alice if Bob holds it, in which case Alice calls $\operatorname{TestDummies}\left(\mathfrak{s}\left(\mathfrak{w}_{\mathrm{t}}\right)\right)$.

2. Alice performs a randomly selected Clifford $C$ on $4 n+2$ qubits jointly on $\mathfrak{s}\left(\mathrm{w}_{c}\right)$ and $\mathfrak{s}\left(\mathrm{w}_{t}\right)$.

3. Alice sends $\mathfrak{s}\left(\mathrm{w}_{c}\right)$ and $\mathfrak{s}\left(\mathrm{w}_{t}\right)$ to Bob; Bob calls TestDummies jointly on them.

4. Alice and Bob perform a TPC whose outcome tells Bob to perform a Clifford unitary $C^{\prime}=\left(K_{c}^{\prime} \otimes K_{t}^{\prime}\right)$ (CNOT) $K^{\dagger}$ where $K$ is the key of the swaddling at this point, and $K_{c}^{\prime}$ and $K_{t}^{\prime}$ are randomly-chosen Cliffords that become the new key.

5. Bob sends $\mathfrak{s}\left(\mathfrak{w}_{c}\right)$ (and $\mathfrak{s}\left(\mathrm{w}_{t}\right)$ if she held this one too) to Alice. Alice calls TestDummies on them.

$\operatorname{RGate}(\mathfrak{s}(\mathrm{w}))$ with $\mathrm{w} \in \mathcal{A}$ :

1. Alice performs a swaddled CNOT with $\mathrm{m}_{w}$ as a control, and $\mathrm{w}$ as target.

2. Alice sends $\mathfrak{s}(\mathrm{w})$ to Bob. Bob calls TestSwaddling $(\mathfrak{s}(\mathrm{w}))$ on it.

3. Alice calls Measure $(\mathfrak{s}(\mathrm{w}))$.

4. Alice and Bob perform a TPC whose result is a Clifford which, if both measurement results were zero, updates the key, and if both measurement results were one, performs a swaddled $e^{i \pi / 4} X P^{\dagger}$ and then updates the key. If the measurement results differ, then they abort.

5. Alice relabels $\mathrm{m}_{a, i}$ to $\mathrm{w}$.

The R-protocol performs the R-gate using gate teleportation[98] via the magic state is very similar to the fault-tolerant version of the R-gate introduced in [7]14]. To perform an R-gate on a wire w via gate teleportation, we would first perform a CNOT from the magic state to $\mathrm{w}$, and then measure $\mathrm{w}$ in the computational basis. If the answer is 0 , we do nothing, and if it is 1 , we perform $e^{i \pi / 4} X P^{\dagger}$ on the former magic state, that we then rename w. Measure $(\mathfrak{s}(\mathrm{w}))$ is described in [5].

\subsection{Security of the Subprotocols}

We will now show that the protocol described in the previous section has the following property: any adversary which deviates significantly from the protocol will be caught cheating with high probability. The general strategy will be as follows. For the initialization, any adversary will be forced to input something into the protocol, and will end up with some state that is properly swaddled. Then, for every other protocol step, we will assume that at the beginning, the inputs are properly swaddled, and will aim to show 
that after the protocol step is done, we are once again left with a correct swaddling of the data, to which the correct operation has been applied. Furthermore, at every step, any deviation from the protocol will be essentially equivalent to an attack on the authentication scheme, which means that an adversary's chances of succeeding in changing the state without getting caught will be negligible in the number of dummy wires per qubit.

\subsection{Some Additional Definitions}

Before we start, we will find it convenient to introduce some additional notation. From now on, $\rho_{0}$ will consist of $\rho_{\text {in }}$ augmented with all additional qubits introduced in the Initialization phase: the additional ancillas, and the dummy wires Alice and Bob use for swaddling. Furthermore, we will denote by $\mathcal{K}_{a}$ and $\mathcal{K}_{b}$ systems which represent Alice's and Bob's current key, respectively. These should be thought of as being part of the inner state of the TPC ideal functionality, and therefore cannot be changed at will. $\mathcal{A}$ represents all other systems at Alice, $\mathcal{B}$ represents all systems in Bob's possession, and $\mathcal{R}$ is a system that includes everything else and ensures that the total state is pure.

Furthermore, in the sequel, we will call a step an execution of any of the subprotocols listed above; each of these steps consists of multiple turns in the sense of Section 2.3 . the state at turn $i$ consists of the state before the $i$ th use of an oracle in the protocol (either a communication oracle or a classical computation oracle).

We will denote by $C_{a, s}$ the operation that encodes Alice's qubits according to the keys stored in the TPC ideal functionalities at turn $s$ in the protocol, and likewise for Bob's encoding operation $C_{b, s}$. See [5] for more precise definitions. Furthermore, we will denote by $[\tilde{\mathscr{A}} \circledast \mathscr{B} \wedge \overline{\mathrm{E}-\mathrm{ABORT}}]_{s}^{\mathscr{O}^{\prime}}\left(\rho_{\mathrm{in}}^{\mathcal{A}_{\text {in }} \mathcal{B}_{\text {in }} \mathcal{R}}\right)$ the global state of the protocol at turn $s$ conditioned on the fact that the protocol doesn't abort before TestAllSwaddlings is completed. Note that this state is not normalized; its trace corresponds to the probability of not aborting before the end.

Definition 5.1 (Forcing). We will say that the protocol is $\mathscr{G} \mathcal{A B}$-forcing for $\tilde{\mathscr{A}}$ at turn $s$ with initial operation $\tilde{\mathscr{E}}_{0} \mathcal{A}$ if there exists a final operation $\tilde{\mathscr{E}}^{\mathcal{A}}$ such that:

$\Delta\left([\tilde{\mathscr{A}} \circledast \mathscr{B} \wedge \overline{\mathrm{E}-\mathrm{ABORT}}]_{s}^{\mathscr{O}^{\prime}}\left(\rho_{\mathrm{in}}^{\mathcal{A}_{\mathrm{in}} \mathcal{B}_{\mathrm{in}} \mathcal{R}}\right), \tilde{\mathscr{E}} \circ \mathrm{C}_{b, s} \circ \mathscr{G} \circ \tilde{\mathscr{E}}_{0}\left(\rho_{\mathrm{in}}^{\mathcal{A}_{\mathrm{in}} \mathcal{B}_{\mathrm{in}} \mathcal{R}}\right)\right) \leqslant \operatorname{negl}(n)$

where $\tilde{\mathscr{E}}_{0}$ is a completely positive, trace non-increasing map that acts only on qubits in Alice's possession at the beginning of the protocol: her own input qubits, the dummies she inputs in the swaddling, and the various ancillas that she adds.

In other words, if the protocol is $\mathscr{G}$-forcing for Alice at some turn $s$, then, if the protocol doesn't abort early, regardless of what she tries to do, it will be essentially equivalent to changing her input (using the initial operation $\tilde{\mathscr{E}}_{0}$ ), executing the operation $\mathscr{G}$ (which will turn out to be the circuit that is supposed to be executed up to turn $s$ ), swaddling the result, and then doing an arbitrary operation on her share alone, represented by $\tilde{\mathscr{E}}$.

Definition 5.2. We say that a subprotocol is $\mathscr{G}$-forcing if the protocol is $\mathscr{G}_{\circ} \mathscr{G}_{0}$-forcing at the end of the subprotocol given that it was $\mathscr{G}_{0}$-forcing at the beginning. 
Definition 5.3 (Hiding). We will say that the protocol is hiding for $\tilde{\mathscr{A}}$ at turn s, if, for all input states $\rho_{\mathrm{in}}^{\mathcal{A}_{\mathrm{in}} \mathcal{B}_{\mathrm{in}} \mathcal{R}_{\mathrm{in}}}$, we have that

$$
\Delta\left(\operatorname{tr}_{\mathcal{B}}\left([\tilde{\mathscr{A}} \circledast \mathscr{B}]_{s}^{\mathscr{O}^{\prime}}\left(\rho_{\mathrm{in}}^{\mathcal{A}_{\mathrm{in}} \mathcal{B}_{\mathrm{in}} \mathcal{R}_{\mathrm{in}}}\right)\right), \operatorname{tr}_{\mathcal{B}}\left([\tilde{\mathscr{A}} \circledast \mathscr{B}]_{s}^{\mathscr{O}^{\prime}}\left(\rho_{\mathrm{in}}^{\mathcal{A}_{\mathrm{in}} \mathcal{R}_{\mathrm{in}}} \otimes|0\rangle\left\langle\left. 0\right|^{\mathcal{B}_{\mathrm{in}}}\right)\right)\right) \leqslant \operatorname{negl}(n)\right.
$$

Hence, the protocol is hiding if the state seen by a dishonest Alice is independent of Bob's input. Note that this in particular implies that $\tilde{\mathscr{A}}$ 's action at turn $s$ is necessarily independent of Bob's input.

\subsection{Proving Hidingness and Forcingness}

To construct the simulator needed to prove security, we will need to show two things. First, we will have to show that before the keys are revealed, the cheater's internal state can be produced by running the protocol internally with a dummy input from the honest party; this will follow from the fact that the Clifford QAS is a secure encryption scheme. Second, we will have to show that after the keys are revealed, the correct circuit has been applied. These two properties correspond to the "hiding" and "forcing" properties defined in the previous section, and proving these for our protocol will be the focus of this section. The fact that the protocol is hiding simply follows from the security of the Clifford QAS as an encryption scheme; we state this as a lemma below. To prove forcingness, the usual trick will be to assume that we pass every call to TestDummies in the protocol (since we only need to look at the no-early-abort case) and use the security definition of the Clifford QAS (Definition 2.2) to show that the dishonest party's attack can be represented by a completely positive, trace non-increasing map $\mathscr{U}^{\text {acc }}$ that acts only on his/her other systems (i.e. the ones that were not involved in the TestDummies). If the adversary decides to try to break the QAS at this step, this $\mathscr{U}^{\text {acc }}$ will simply decrease the trace to reflect the probability of abortion. We prove the forcingness of the various subprotocols in [5], and simply summarize the end result as Lemma 5.5]below.

Lemma 5.4. For every turn before OpenAllSwaddlings, the protocol is hiding for every adversary $\tilde{\mathscr{A}}$ and $\tilde{\mathscr{B}}$.

Proof. During this phase of the protocol, all $\tilde{\mathscr{A}}$ ever gets from Bob (and $\tilde{\mathscr{B}}$ from Alice) is encrypted in a QAS, whose key is managed by the classical two-party computations. Hence, this follows directly from the security of the Clifford QAS (see Section 2.2, or the full version [5] for more details).

Lemma 5.5. All subprotocols are $\mathscr{G}$-forcing for any $\tilde{\mathscr{A}}$ and $\tilde{\mathscr{B}}$, where $\mathscr{G}$ is the operation performed by the subprotocol.

\section{Proving Active Security}

Here is the rough description of $\tilde{\mathscr{C}}$ 's operations for the start of the simulation. These steps allow to simulate any execution aborting in the real world before the adversary $\tilde{\mathscr{A}}$ receives back all her swaddlings from $\mathscr{B}$ in TestAllSwaddlings. Let $s^{*}$ be the turn in $\widehat{\Pi}_{\mathscr{F}}^{\mathscr{O}^{\prime}}$ at which this transmission is received by $\tilde{\mathscr{A}}$. The simulator $\tilde{\mathscr{C}}$ runs $\tilde{\mathscr{A}}$ as a subroutine using an internal copy of $\mathscr{B}$ 's instructions run upon a (or any) dummy input state $|0\rangle^{\mathcal{B}_{\text {in }}}$. We denote by $\mathscr{B}^{*}$ the simulated $\mathscr{B}$ on a dummy input run internally in $\tilde{\mathscr{C}}$. 
We define ${\mathrm{E}-\mathrm{ABORT}_{s}}_{s}$ as the event consisting in an execution between two parties aborting at turn $s<s^{*}$. Let $\left[\tilde{\mathscr{C}} \circledast \mathscr{D} \wedge \mathrm{E}-\mathrm{ABORT}_{s}\right]^{\mathscr{F}}\left(\rho_{\text {in }}\right)$ and $\left[\tilde{\mathscr{A}} \circledast \mathscr{B} \wedge \mathrm{E}-\mathrm{ABORT}_{s}\right]^{\mathscr{O}^{\prime}}\left(\rho_{\text {in }}\right)$ be denoting the the joint state of an execution that aborts at turn $s$ upon joint input state $\rho_{\text {in }}$ between $\tilde{\mathscr{C}}$ and $\mathscr{D}$ (in the ideal world) and between $\tilde{\mathscr{A}}$ and $\mathscr{B}$ (in the real world) respectively. States $\left[\tilde{\mathscr{C}} \circledast \mathscr{D} \wedge \mathrm{E}-\mathrm{ABORT}_{s}\right]^{\mathscr{F}}\left(\rho_{\text {in }}\right)$ and $\left[\tilde{\mathscr{A}} \circledast \mathscr{B} \wedge \mathrm{E}-\mathrm{ABORT}_{s}\right]^{\mathscr{O}^{\prime}}\left(\rho_{\text {in }}\right)$ are not normalized, $\operatorname{tr}\left(\left[\tilde{\mathscr{C}} \circledast \mathscr{D} \wedge \mathrm{E}-\mathrm{ABORT}_{s}\right]^{\mathscr{F}}\left(\rho_{\text {in }}\right)\right)$ and $\operatorname{tr}\left(\left[\tilde{\mathscr{A}} \circledast \mathscr{B} \wedge \mathrm{E}-\mathrm{ABORT}_{s}\right]^{\mathscr{O}^{\prime}}\left(\rho_{\text {in }}\right)\right)$ are the probabilities that $[\tilde{\mathscr{C}} \circledast \mathscr{D}]^{\mathscr{F}}\left(\rho_{\text {in }}\right)$ and $[\tilde{\mathscr{A}} \circledast \mathscr{B}]^{\mathscr{O}^{\prime}}\left(\rho_{\text {in }}\right)$ aborts at step $s$ respectively. The proof of next lemma can be found in [5] and follows easily from the hiding property of the protocol expressed in Lemma 5.4

Lemma 6.1 (Early abort). Let $\tilde{A}$ be an adversary in hybrid protocol $\widehat{\Pi}_{\mathscr{F}}^{\mathscr{O}^{\prime}}=$ $\left(\mathscr{A}, \mathscr{B}, \mathscr{O}^{\prime}, m\right)$ for $\mathscr{F}: \mathrm{L}\left(\mathcal{A}_{\text {in }} \otimes \mathcal{B}_{\text {in }}\right) \rightarrow \mathrm{L}\left(\mathcal{A}_{\text {out }} \otimes \mathcal{B}_{\text {out }}\right)$. Let $s^{*}$ be the turn in $\widehat{\Pi}_{\mathscr{F}}^{\mathscr{O}^{\prime}}$ at which $\mathscr{B}$ returns all of $\tilde{\mathscr{A}}$ 's swaddlings in TestAllSwaddlings and let $0 \leq s<s^{*}$. Then, there exists an adversary $\tilde{\mathscr{C}}$ in $\Gamma_{\mathscr{F}}$ (polysize in the size of $\left.\tilde{\mathscr{A}}\right)$ such that for any $\rho_{\text {in }} \in \mathrm{D}\left(\mathcal{A}_{\text {in }} \otimes \mathcal{B}_{\text {in }} \otimes \mathcal{R}\right)$,

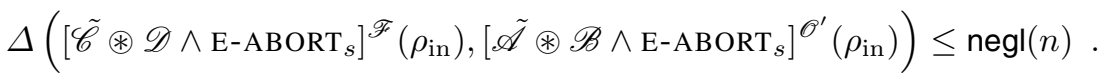

By symmetry, the same is also true with respect to adversaries $\tilde{\mathscr{B}}$ in $\widehat{\Pi}_{\mathscr{F}}^{\mathscr{O}^{\prime}}$ and $\tilde{\mathscr{D}}$ in $\Gamma_{\mathscr{F}}$.

It remains to simulate the execution from turn $s^{*}$ until the end. In the simulated world, if $\tilde{\mathscr{C}}$ reaches turn $s^{*}$ when $\tilde{\mathscr{A}}$ and $\mathscr{B}^{*}$ are interacting then the output state $\mathscr{F}\left(\rho_{\text {in }}^{\mathcal{A}_{\text {in }} \mathcal{R}} \otimes\right.$ $|0\rangle\langle 0|)$ can be recovered. The reason being that at turn $s^{*}$, all swaddlings have been tested by $\mathscr{B}^{*}$ in TestAllSwaddlings. $\tilde{\mathscr{C}}$ can get the output state since it knows all keys allowing to decrypt all logical wires, and all these wires have not been tampered with. The simulation then works along the same lines than in [4]. At turn $s^{*}, \tilde{\mathscr{C}}$ is simulating $\mathscr{B}^{*}$ 's quantum transmission of all swaddlings belonging to $\tilde{\mathscr{A}}$ back to $\tilde{\mathscr{A}}$. These swaddlings have been successfully tested by $\mathscr{B}^{*}$ in TestAllSwaddlings. $\tilde{\mathscr{C}}$ intercepts all these swaddlings and decrypts them together with all swaddlings held by $\mathscr{B}^{*} . \tilde{\mathscr{C}}$ then recovers the output state. $\tilde{\mathscr{C}}$ undoes the quantum operation $\mathscr{F}$ in order to recover $\tilde{\mathscr{A}}$ 's effective input state before querying $\mathscr{F}_{1}$ with the effective input state. $\tilde{\mathscr{C}}$ swaddles back the answer to the query using the same keys. The swaddlings are finally sent to $\tilde{\mathscr{A}}$ before resuming the interaction between $\tilde{\mathscr{A}}$ and $\mathscr{B}^{*}$. Two things can happen in OpenAllSwaddlings: 1) $\tilde{\mathscr{A}}$ 's behavior makes the execution abort, and 2) $\tilde{\mathscr{A}}$ and $\mathscr{B}$ reach the end of the execution in normal conditions. In the first case, $\tilde{\mathscr{A}}$ may even get the output state of the computation while preventing $\mathscr{B}$ from recovering his own. When the output state is available to $\tilde{\mathscr{A}}, \tilde{\mathscr{C}}$ will need to call $\mathscr{F}_{1}$ (with $\tilde{\mathscr{A}}$ 's effective input) and $\mathscr{F}_{2}$ where the later call is without fairness (i.e., $f=0$ ) when $\tilde{\mathscr{A}}$ prevents $\mathscr{B}$ from decrypting his output logical wires.

Let $\overline{\mathrm{E}-\mathrm{ABORT}}$ be the event of not having E-ABORT ${ }_{s}$ at any turn $s<s^{*}$. Next lemma establishes the active security when no early aborting occurs. The proof can be found in [5]. It follows from the forcingness of the protocol established in Lemma 5.5

Lemma 6.2 (No early abort). For any quantum adversary $\tilde{\mathscr{A}}$ in hybrid protocol $\widehat{\Pi}_{\mathscr{F}}^{\mathscr{O}^{\prime}}=\left(\mathscr{A}, \mathscr{B}, \mathscr{O}^{\prime}, m\right)$ for $\mathscr{F}: \mathrm{L}\left(\mathcal{A}_{\text {in }} \otimes \mathcal{B}_{\text {in }}\right) \rightarrow \mathrm{L}\left(\mathcal{A}_{\text {out }} \otimes \mathcal{B}_{\text {out }}\right)$, there exists an adversary $\tilde{\mathscr{C}}$ in $\Gamma_{\mathscr{F}}$ (polysize in the size of $\tilde{\mathscr{A}}$ and $\mathscr{B}$ ) such that for any $\rho_{\mathrm{in}} \in$ $\mathrm{D}\left(\mathcal{A}_{\text {in }} \otimes \mathcal{B}_{\text {in }} \otimes \mathcal{R}\right)$ 


$$
\left.\left.\Delta([\tilde{\mathscr{C}} \circledast \mathscr{D} \wedge \overline{\mathrm{E}-\mathrm{ABORT}}]]^{\mathscr{F}}\left(\rho_{\mathrm{in}}\right),[\tilde{\mathscr{A}} \circledast \mathscr{B} \wedge \overline{\mathrm{E}-\mathrm{ABORT}}]\right]^{\mathscr{O}^{\prime}}\left(\rho_{\mathrm{in}}\right)\right) \leq \operatorname{negl}(n)
$$

By symmetry, the same is also true with respect to adversaries $\tilde{\mathscr{B}}$ in $\widehat{\Pi}_{\mathscr{F}}^{\mathscr{O}^{\prime}}$ and $\tilde{\mathscr{D}}$ in $\Gamma_{\mathscr{F}}$.

Since Lemmas 6.1 and 6.2 together show that a simulator succeeds in reproducing any real execution, we conclude the active security of the protocol:

Theorem 6.3 (Active security). For any polynomial-time quantum operation $\mathscr{F}$ : $\mathrm{L}\left(\mathcal{A}_{\text {in }} \otimes \mathcal{B}_{\text {in }}\right) \rightarrow \mathrm{L}\left(\mathcal{A}_{\text {out }} \otimes \mathcal{B}_{\text {out }}\right)$, the two-party hybrid protocol $\widehat{\Pi}_{\mathscr{F}}^{\mathscr{O}^{\prime}}$ is statistically active secure without fairness for Bob.

\section{References}

1. Aharonov, D., Ben-Or, M., Eban, E.: Interactive proofs for quantum computations. In: Proceedings of Innovations in Computer Science (2008), http: //arxiv.org/abs/0810.5375

2. Barnum, H., Crépeau, C., Gottesman, D., Smith, A., Tapp, A.: Authentication of quantum messages. In: 43rd Annual IEEE Symposium on Foundations of Computer Science (FOCS), pp. 449-458 (2002)

3. Bravyi, S., Kitaev, A.: Universal quantum computation with ideal clifford gates and noisy ancillas. Physical Review A 71, 022316 (2005), quant-ph/0403025

4. Dupuis, F., Nielsen, J.B., Salvail, L.: Secure Two-Party Quantum Evaluation of Unitaries against Specious Adversaries. In: Rabin, T. (ed.) CRYPTO 2010. LNCS, vol. 6223, pp. 685706. Springer, Heidelberg (2010)

5. Dupuis, F., Nielsen, J.B., Salvail, L.: Actively secure two-party evaluation of any quantum operation. Cryptology ePrint Archive, record 2012/304 (2012), http://eprint.iacr.org/

6. Gottesman, D.: Stabilizer codes and quantum error correction. PhD thesis, California Institute of Technology (1997)

7. Gottesman, D.: An introduction to quantum error correction and fault-tolerant quantum computation. In: Lomonaco Jr., S.J. (ed.) Quantum Information Science and Its Contributions to Mathematics. Proceedings of Symposia in Applied Mathematics, vol. 68, pp. 13-60 (April 2010), http: / /arxiv.org/abs/0904.2557

8. Gottesman, D., Chuang, I.L.: Demonstrating the viability of universal quantum computation using teleportation and single-qubit operations. Nature 402, 390-393 (1999)

9. Gottesman, D., Chuang, I.L.: Quantum teleportation is a universal computational primitive (August 1999), http: / / arxiv.org/abs / quant-ph/9908010

10. Gutoski, G., Watrous, J.: Toward a general theory of quantum games. In: 39th Annual ACM Symposium on Theory of Computing (STOC), pp. 565-574 (2007)

11. Hallgren, S., Smith, A., Song, F.: Classical Cryptographic Protocols in a Quantum World. In: Rogaway, P. (ed.) CRYPTO 2011. LNCS, vol. 6841, pp. 411-428. Springer, Heidelberg (2011)

12. Lunemann, C., Nielsen, J.B.: Fully Simulatable Quantum-Secure Coin-Flipping and Applications. In: Nitaj, A., Pointcheval, D. (eds.) AFRICACRYPT 2011. LNCS, vol. 6737, pp. 21-40. Springer, Heidelberg (2011)

13. Regev, O.: On lattices, learning with errors, random linear codes, and cryptography. In: 37 th Annual ACM Symposium on Theory of Computing (STOC), pp. 84-93 (2005)

14. Shor, P.W.: Fault-tolerant quantum computation. In: 37th Annual IEEE Symposium on Foundations of Computer Science (FOCS), pp. 56-65 (1996) 